\section{Impacto socio-espacial de la competitividad de las Mipymes turísticas situadas en el Parque Nacional Puyehue y su área de influencia*}

Socio-spatial impact of competitiveness in touristic MSMEs located in the Puyehue National Park and its surroundings

Claudio Rosales U. * Rodolfo Lemarie O. **

Esta investigación es el resultado del proyecto de investigación DIULA N N 12/2012 "Análisis de las disparidades económicas, sociales y espaciales de las PYMES del Sector Turismo: Caso de estudio - Parque Nacional Puyehue" y del Proyecto Fondecyt $N^{\circ} 1140936$ denominada "Relaciones Político Administrativas y su Funcionalidad para el Último Lustro en el Eje Longitudinal de la Vertiente de Barlovento de la Cordillera de Los Andes Norpatagónicos".

** Profesor asociado, área de Geografía Económica y Regional, Departamento de Ciencias Sociales, investigador del Núcleo de Economía Regional de la Universidad de los Lagos, Osorno Chile. crosales@ulagos.cl.

*** Profesor Asociado, área TICs y Gestión Informática. Departamento de Ciencias Administrativas y Económicas. Investigador Núcleo de Economía Regional de la Universidad de los Lagos, Osorno Chile. rlemarie@ulagos.cl

\section{Resumen}

Esta investigación tuvo por objeto estudiar el impacto socio-espacial de la competitividad existente en micros y pequeños empresarios vinculados al turismo local, en la localidad de Entre Lagos, como asimismo en aquellos localizados desde este centro poblado hacia los ejes de contacto espacial al Parque Nacional Puyehue, como son las rutas Entre Lagos - Mantilhue (T985); Entre Lagos - Anticura $(\mathrm{CH}-215)$ y Entre Lagos - El Encanto (U-475). El estudio permitió confirmar el escaso nivel de competitividad que estos emprendedores presentan. La afirmación de esta tesis se logró a partir de la aplicación del método de Castaño y Gutiérrez (2011). Los resultados arrojaron que 3 de los 8 factores de la metodología, explicaron más del $62 \%$ de la baja competitividad. Estas fueron las funciones: Administrativa, Comercial / Logística y Talento Humano. En consecuencia, la participación de estos factores serán claves para revertir la actual situación que afecta a estos actores, quienes perciben en el turismo local un motor de desarrollo a sus precarias condiciones económicas.

Palabras clave: Impacto socio-espacial, Competitividad, Mipymes, Turismo, Parque Nacional Puyehue

\begin{abstract}
The present investigation was designed to study the social and spatial impact of competitiveness in micro and small entrepreneurs linked to local tourism in Entre Lagos and, also locations that are between the routes that connect to the Puyehue National Park to the town previously mentioned, such as the routes Entre Lagos
\end{abstract}


- Mantilhue (T-985); Entre Lagos - Anticura $(\mathrm{CH}-215)$ and Entre Lagos - Encanto (U-475). The study allowed to confirm the low level of competitiveness that these entrepreneurs exhibit. The assertion of the thesis was achieved by applying the method of Castaño \& Gutierrez (2011). The results allowed to say that 3 of the 8 factors of the methodology, explained more than $62 \%$ of low competitiveness. These were the functions: administrative, commercial / logistics and human talent. Consequently, improving these factors is key in order to reverse the current situation of these actors, who perceive that local tourism is a development opportunity in their precarious economic conditions.

Key words: Socio-spatial impact, Competitiveness MSMEs, Tourism, Puyehue National Park

\section{Introducción}

La presente investigación analizó los impactos socio-espaciales de la competitividad de las mipymes formales localizadas en las comunas de Río Bueno y Puyehue, a partir de los ejes de contacto espacial al PNP como son los circuitos asociados a la rutas Entre Lagos - Mantilhue (T985); Entre Lagos - Anticura (CH-215) y Entre Lagos - El Encanto (U-475).ancho por 27.94 centímetros de alto, márgenes estándar.

Para tal efecto, el objetivo central está orientado al estudio de estas micro, pequeñas y medianas empresas (MIPYMES) turísticas, con el objeto de establecer aquellos factores sociales y económicos más determinantes del nivel de competitividad, presentes en cada uno de ellos, y complementariamente establecer de la manera más fidedigna otros factores de naturaleza espacial que también condicionan la compleja realidad que actualmente afectan a estos emprendedores turísticos.

Para abordar la investigación se utilizó información tanto secundaria como primaria: la primera, obtenida a partir de los registros de emprendimientos con que cuenta el Servicio Nacional de Turismo (SERNATUR, 2014) y las Ilustres Municipalidades de Río Bueno y Entre Lagos; la segunda, de génesis primaria, obtenida mediante el trabajo de campo realizado en Entre Lagos y cada uno de los 3 ejes viales citados. Bajo el análisis de estas dos fuentes, fue posible contrastar la información oficial versus la disponible en terreno, como asimismo georeferenciarla e ilustrarla en una cartografía addoc mediante el uso del software libre QGIS 2.8. Posteriormente, el análisis de la competitividad se realiza a través de un instrumento de medición validado por Castaño y Gutiérrez, (2011) 1 . El modelo utilizado consta de 8 factores, denominados como: Función Gerencial, Función Administrativa, Función Comercial y Logística, Función Financiera, Talento Humano, Función Tecnológica, Función Ambiental y Factores Externos. Cabe consignar que cada factor tiene variables que los miden, los cuales suman un total de 38 , los que dan cuenta de los componentes asociados a cada factor. Con esto fue posible no solamente medir el peso relativo de cada uno de ellos, sino que

Debemos consignar que la mayor parte de los modelos que intentan medir la competitividad, están orientados principalmente hacia las grandes empresas y dirigidos a países del mundo desarrollado. En este sentido, tomando en consideración lo citado, se optó por esta metodología, por cuanto en primer lugar ha sido aplicado a realidades de naciones menos desarrolladas, a ámbitos espaciales más locales y de naturaleza más rural, como así también a emprendedores pequeños y medianos, como son lo que nos interesa analizar en esta investigación. 
también poder hacer una evaluación de todo el sistema en su globalidad.

Finalmente, la estructura del presente artículo se conforma por tres capítulos. El primero, se orienta a realizar una revisión temporoespacial respecto a cómo se dio el proceso de transformación económica en Chile a partir de la década de los ochenta en adelante; para luego centrarnos en procurar una aproximación conceptual respecto al tema de esta investigación, como es el de la competitividad. El segundo Capítulo aborda las actividades turísticas asociadas tanto al Parque Nacional Puyehue como al área de influencia a dicha unidad. Paralelamente, se procede a identificar, caracterizar y dimensionar los emprendimientos turísticos formales existentes en Entre Lagos, y en cada uno de los ejes viales consignados en el estudio. Por último, el tercer capítulo, entrega los resultados que se obtienen a partir de la aplicación del método empleado por Castaño \& Gutiérrez, respecto al nivel de competitividad de estos micro y pequeños empresarios formales. Este análisis además será complementado de manera cualitativa con aquellos emprendedores informales.

\section{Capítulo I}

Desde que Chile, en la década de los ochenta del siglo pasado, asumió en un contexto político dictatorial en plenitud un modelo de desarrollo económico basado en el libre mercado orientado fundamentalmente a las exportaciones no tradicionales, era dable esperar importante cambios en lasociedad. En efecto, parafraseando a Rosales (1990), las transformaciones en esta materia se manifestaron en un conjunto de acciones que se expresaron, entre otras, en: cambio en la política cambiaria (dólar), rebajas arancelarias (aduanas), creación de Pro-Chile, modificación al impuesto a la compraventa por el IVA, franquicias de orden legal otorgadas a las Ilegadas de capitales externos, eliminación de trabas administrativas a la gestión pública, etc.

Lo anterior, pronto comenzó a tener réditos en materia económica que se expresaron no solamente con la llegada capitales externos, sino que también, en la medida que se iba avanzando en este proceso, se comenzaron a producir "efectos de demostración" en nuevos emprendedores, quienes vieron una salida a su menguada situación económica.

La puesta en escena de estas nuevas políticas económicas dieron inicio a un proceso que afectó fuertemente tanto el nivel institucional como el privado, sin precedente en la historia reciente de Chile. En efecto, durante estas primeras décadas, este país, eminentemente minero en materia de exportación, ahora comienza a convivir con otras actividades vinculadas al ámbito agroalimentario, como son la industria de la fruticultura y acuicultura principalmente.

El rol que comienzan a tener tanto los actores privados como públicos en este contexto exportador serán diversos y complementarios, por cuanto debieron ambos tener que conjugar no solo las políticas públicas que se diseñaron para estos fines, sino que también articular los sistemas financieros y aquellas dimensiones que tocaban ámbitos más locales, donde la historia socio-demográfica y territorial pasaron a constituirse en disparadores claves para iniciar estos nuevos emprendimientos, de acuerdo a como lo exigían los mercados demandantes más desarrollados del hemisferio norte. 
Tuvieron que pasar varios años para que surgiera en este sistema exportador un tercer actor, representado en este caso por la comunidad. Al respecto, un factor que fue determinante, en cuanto a visibilizar esta participación ciudadana más organizada, ocurre con posterioridad a la llegada de la democracia. Poco a poco comienzan a aparecer organizaciones sociales -especialmente ambientales- que se inician y denuncian ciertos procesos productivos que se desarrollaban a un costo social y ambiental muy alto.

Hemos considerado pertinente hacer hincapié en estos hechos, por cuanto serán claves para comprender al menos dos situaciones que explican en parte el marco de este trabajo. El primero se registra de manera incipiente a fines de la década de los noventa y con mayor fuerza a partir del siglo XXI, en cuanto a la necesidad de tener que incorporar mayor valor agregado a los productos que se exportan, hecho conocido en la jerga económica como el tránsito hacia una segunda fase exportadora. El segundo aspecto se presenta casi paralelamente con esta necesidad, como fue la de explorar y abrir nuevas alternativas de emprendimiento orientadas a diversificar la economía. Es así como, en este contexto, surge como una de ellas el turismo asociado a intereses especiales, donde el ecoturismo, el agroturismo y el etnoturismo aparecen como símbolos de esta nueva manera de relacionarse con el entorno, más amigable y de menor impacto ambiental a los ya dañados ecosistemas naturales y culturales, tales como los que están presentes en las regiones sur australes de Chile.

El articular esta nueva ecuación productiva, competitividad v/s turismo, no ha sido sencilla. Muy por el contrario, los desafíos que demanda el hacer del turismo una actividad más competitiva y con mayor valor agregado pone a todos los actores de la sociedad, y particularmente a los privados, en una posición donde se tendrán que redefinir y precisar la ya clásicas interrogantes económicas del ¿Qué?, el ¿Dónde?, el ¿Cómo? y el ¿Cuándo producir?, entre otras.

Para aproximarnos a esta reflexión es primordial aportar con algunos antecedentes que nos permitan puntualizar cómo se concibe la competitividad, insumo necesario y central para comprender las etapas siguientes que examinaremos empíricamente en esta investigación.

Para comenzar, diremos que la competitividad, como concepto, no tiene límites precisos y se define en relación con otros conceptos. El más cercano, según la Real Academia de la Lengua (2014), es el de competencia, palabra que viene del latín competentia. Sin embargo, esta expresión, sin asignarle ningún adjetivo calificativo, se puede concebir como disputa o pugna entre dos personas o sobre un determinado objeto, interés o cuestión. También se la define, desde el ámbito más del derecho, como la capacidad de intervenir en un determinado tema o asunto.

Atendiendo lo citado precedentemente, podemos consignar que si deseamos aproximarnos gradualmente a puntualizar la competitividad en términos económicos, nos encontraremos como es dable esperar con diversas acepciones. En efecto, desde un sentido más purista, esta se entiende como la concurrencia de varios actores en el mercado, siendo el objetivo obtener beneficios económicos. El mundo de la economía reconoce varios tipos de competencia. Entre 
ellas destaca la competencia perfecta, es decir, quienes ofrecen y demandan productos y servicios en el mercado no deben tener influencia sobre los precios, por cuanto este debe regularse según la demanda. También se reconoce la competencia imperfecta. Esta hace referencia a aquellas situaciones en las cuales hay quienes tienen poder para influir en los precios, ya sea por parte de los oferentes o de los demandantes, generándose situaciones de monopolio, oligopolio etc.

No obstante, si queremos en esta materia transitar desde la concepción de la competencia al de la competitividad, necesariamente tendremos que hacer mención a quienes dieron origen a este segundo concepto. En tal sentido, uno de los precursores de los tiempos más actuales es sin duda Michael Porter (1985). Para él, la competitividad se concibe como: "la capacidad de producir bienes utilizando los recursos humanos, económicos y naturales disponibles de la manera más eficaz y rentable posible". Sobre la base de esta definición, indica que para una empresa, el valor más importante de competitividad está dado por la capacidad de generar en sus clientes mejores precios respecto de sus competidores y contar con productos diferenciados, cuyos ingresos superen los costos de producción. Prosigue señalando que, para alcanzar estos objetivos, es necesario diseñar una "estrategia competitiva" que contempla cinco grandes fuerzas que se deben controlar para sobrevivir al mercado y tomar buenas decisiones. Estas son: la llegada de nuevos competidores y de productos sustitutos, la capacidad de negociación con los proveedores y clientes, y por último, la rivalidad entre competidores. Finalmente, aduce que actualmente se está agregando una sexta fuerza dada por el Gobierno (actor gubernamental), quien puede regular el mercado e incluso competir con el mundo privado, hecho que a la luz tanto de la teoría como de la praxis aún no está resuelto.

Así como Porter ha hecho una importante contribución, a partir de la mitad del siglo $X X$, debemos consignar que también otros pensadores ya habían estado reflexionando al respecto. En este sentido, es dable indicar que ya los filósofos griegos, entre los cuales destaca Aristóteles (330 AC), quien en el su libro "La Política Capítulo IV" y en un lenguaje de la época, nos planteaba que "los verdaderos valores de la riqueza radicaba en la naturaleza de las cosas, en el lugar donde se encuentran, en su utilidad relativa y la manera de emplearlos, como asimismo, en determinar que especies son más provechosas y cuales convienen desarrollar en cada localidad". Con esto queremos plasmar que muchos de los conceptos que actualmente son de uso común en la ciencias económicas y sociales para abordar estas temáticas, ya fueron hace muchos siglos concebidos e internalizados por este pensador griego.

Varios siglos después, en el siglo XVIII aparece otro clásico, como Adam Smith (1776), quien nos relataba que bajo un sistema económico de libre cambio, cada nación debe especializarse en producir esas mercancías que podrían ser lo más eficientemente posible. Algunos de éstos serían exportados a la paga para las importaciones de las mercancías que se podrían producir más eficientemente a otra parte. Al respecto, indica que el gran elemento diferenciador se sustenta en la especialización, la cual permite minimizar los costos absolutos y con ello maximizar el beneficio. Concluye que esta debiera ser la vía para que un país obtenga mayores ganancias y que, paralelamente, el comercio se convierta 
en el generador de crecimiento en la producción mundial. Del mismo modo, otro contemporáneo a él, David Ricardo (1817), avanza aún más en la teoría planteada por Smith, estableciendo que los costos relativos son más importantes que los absolutos para determinar la ventaja entre los países. Esto lo respalda en que en ellos es posible marcar la diferencia, por cuanto están más sujetos a los cambios relacionados con las tecnologías, la innovación, entre otros.

En tiempos más actuales, también hemos hallado otras definiciones, como la elaborada por la Comisión de las Comunidades Europeas (2003), quienes acordaron, en lo que respecta a la competitividad industrial, definirla como una medida en la cual se determina la capacidad inmediata y futura del sector industrial para diseñar, producir y vender bienes cuyos atributos logren formar un paquete más atractivo que el de productos similares ofrecidos por los competidores, siendo el juez final el mercado. Tomando parte de esta definición, Haguenauer (1990) la define como la capacidad de una industria o empresa para producir bienes con patrones de calidad específicos, utilizando más eficientemente recursos que empresas 0 industrias semejantes en el resto del mundo, durante un cierto período de tiempo.

También en este devenir de reflexiones, han surgido intentos por humanizar un poco más el concepto. Es así como, a través del President's commission on industrial competitiveness(1985), nos indicaban que bajo condiciones de mercado libre, se deben producir bienes y servicios que satisfagan no solo los requerimientos de los mercados internacionales, sino que también debiera ser posible mantener o expandir los ingresos reales de sus ciudadanos. De lo anterior, se evidencia que para alcanzar una posición competitiva se requiere, entre otras cosas: la incorporación de progreso técnico, entendido como la capacidad de imitar, adaptar y desarrollar técnicas de producción de bienes y servicios antes inexistentes en una economía o de su mejoramiento; la diversificación de los productos exportables en condiciones de calidad y precio al menos equiparables a las de sus competidores; la adaptación a las nuevas condiciones de competencia en los mercados y la reconversión de sectores no competitivos.

En este análisis, un poco más pormenorizado sobre la competitividad económica, no podemos dejar fuera, dado el carácter socio-espacial de la presente investigación, las concepciones referidas a la competitividad territorial. Al respecto, no son pocos los autores que en la actualidad nos están planteando concepciones que apuntan a la lógica de hacer "competir a los territorios". En este contexto, según lo planteado por el Observatorio Europeo Leader (1997), se nos indica en términos generales que la competitividad territorial adquiere un carácter competitivo si puede afrontar la competencia del mercado y garantizar al mismo tiempo la viabilidad medio-ambiental, económica, social y cultural, aplicandológicas de red y dearticulación interterritorial. Por otra parte, parafraseando a Porter (1991) y Krugman (1992), en estricto rigor los territorios no compiten perse, sino que son las empresas las que lo hacen; por lo tanto, un país lo hacen competitivo las empresas competitivas que hay en este y no su territorio.

Con lo citado precedentemente queremos ilustrar una cuestión que permanentemente está en la discusión académica, respecto a la existencia de regiones ganadoras y perdedoras ante este mundo globalizado e internacionalizado, tal cual lo plantean Szary (1997) y Benko y Lipietz 
(1992). Si bien es posible constatar, a partir de estos estudios, territorios mejor posicionados que otros, estimamos que esta situación se presenta básicamente en términos de lo que son las "ventajas comparativas", tal cual ocurre con muchos países subdesarrollados o en vías de desarrollo. Es decir, muchos de estos territorios presentan valiosos e importantes recursos naturales, lo que les otorga una condición potencial enorme en esta materia. Sin embargo, no podemos indicar lo mismo en cuanto a lo que son las "ventajas competitivas", por cuanto a diferencia de la anterior, las primeras están dadas, en cambio las segundas deben crearlas. Esto es lo que finalmente ha estado marcando las grandes brechas socio-económicas que observamos entre el mundo desarrollado y el sub-desarrollado.

Como es posible apreciar, hemos intentado ilustrar que no han sido pocos los intentos que se han realizado para conceptualizar de manera más precisa y concreta la competitividad, tanto económica como territorial. No obstante, lo que queda de manifiesto es que no existe una mirada única; muy por el contrario, tal como finalmente los mismos autores y organizaciones aludidas lo indican, existiría al parecer una complementariedad de elementos, donde la combinación de estos atenuarían los principios más clásicos de la competencia perfecta, como son los rendimientos a escala constantes y la ausencia de externalidades. En consecuencia, podemos desprender que la competitividad no es producto de una casualidad ni surge espontáneamente; se crea y se logra a través de un largo proceso de aprendizaje y negociación por grupos colectivos representativos que configuran la dinámica de conducta organizativa, como los accionistas, directivos, empleados, acreedores, clientes, por la competencia y el mercado y por último, el gobierno y la sociedad en general.

Pasando a un escenario más local, con el pasar de los años se ha hecho cada vez más difícil para estas economías emergentes como lo ha sido la chilena hacer este giro. El hecho más notable lo constituye el crecimiento presentado entre los años 1986 a 1997, donde todos los indicadores de producción registrados por el Banco Central de Chile, como asimismo por instituciones internacionales, posicionaban al país como un ejemplo a seguir².

Durante este periodo de bonanza, el crecimiento económico se explicó en gran medida por la explotación de las ventajas comparativas, las cuales jugaron un rol clave para explicar las positivas tasas obtenidas.

Sin embargo, este proceso auspicioso ya en aquellos años manifestaba claramente la necesidad de avanzar en la generación de incorporar mayor valor agregado a lo que se estaba produciendo. Además, este no era un tema desconocido para el país, por cuanto se tenían antecedentes no solo atribuible a la historia económica de estas naciones más desarrolladas, sino que también eran referentes muy cercanos, dado a que muchos de ellos eran nuestros mercados demandantes.

En este contexto, pasar a una segunda fase exportadora, con mayor valor agregado resulta primordial para cualquier país, debido a que esto

\footnotetext{
La década de oro de crecimiento económico que ha tenido Chile se extendió entre 1986-1997. En efecto, durante estos años Chile creció a tasas cercanas a 7\% real anual, lo que significó que, en términos nominales, se pasara de un PIB de cerca de US $\$ 38,000$ millones en 1986 a un poco más de US\$77,000 millones en 1997.
} 
implica invertir fuertemente en capital humano avanzado, innovación, tecnología, entre otros ${ }^{3}$. Debemos consignar que este proceso en Chile, cada vez fue quedando más en evidencia, por cuanto el país, al margen de comercializar, fue firmando una serie de tratados de libre comercio, los cuales si bien nos garantizaba una mayor estabilidad con este mundo más desarrollado en materia de exportación, también nos colocaba exigencias mayores, principalmente en términos de compromisos con la economía de mercado, como así también se nos exigía el mantener una estabilidad política basada en sistemas de naturaleza democrática ${ }^{4}$.

En consecuencia, el que Chile esté interactuando preferentemente con estas naciones más desarrolladas, si bien es una oportunidad, también es un gran desafío. En efecto, este hecho nos obliga a tener que cumplir a cabalidad no solamente con los compromisos adquiridos, sino que también a desarrollar procesos de trazabilidad productiva cada vez más exigentes.

Por lo tanto, resulta algo preocupante, si observamos la situación de Chile de estos últimos años. Según el Word Economic Forum, para el año 2015, nuestro país ocupó el lugar $N^{\circ}$

Datos que ilustran esta situación se aprecian en los actuales índices de competitividad a nivel mundial, lo cuales son liderados por países como: Suiza, Singapur, Estados Unidos y Alemania (Word Economic Forum, 2015).

Según la Dirección General de Relaciones Económicas Internacionales de Chile, actualmente existen tratados de libre comercio con los principales mercados del mundo, alcanzando más de 4.200 millones de personas distribuidas en los cinco continentes. Hasta la fecha (2015) se han suscrito 24 acuerdos comerciales con 63 países. Además, Chile participa en organismos internacionales como: APEC, OCDE, OMC, OMPI, WWTG y últimamente con el TPP.
35 a nivel mundial en competitividad, más allá que sea el mayor de América Latina. No obstante, este ranking, si lo examinamos temporalmente, está mostrando una baja, por cuanto para el año 2014 el país ocupaba el lugar 33, es decir, hemos estado perdiendo competitividad. Este hecho se correlaciona con lo informado por el Banco Mundial, el cual proyecta que la economía nacional se expandirá 2,4\% en 2016 , frente a la estimación de 3,3\% anunciada en junio. Para 2017, la entidad augura un alza de $2,9 \%$, lo que se compara con el vaticinio previo de $3,5 \%$, mientras que para 2018 pronostica un avance de $3,1 \%$.

Sobre la base de lo citado precedentemente, y ante los vaivenes naturales del mercado, no nos debiera sorprender que Chile debe avanzar en diversificar su economía, de tal manera de irse desprendiendo gradualmente de la ya clásica explotación de materias primas con escaso valor agregado. Esto implicará necesariamente mejorar la competitividad en el más amplio sentido del concepto. Es en este marco económico mundial y nacional de nuestras exportaciones donde está apareciendo altamente valorizado el turismo (SERNATUR 2011), respecto de aquellas actividades más vinculadas a la explotación de materias primas, como las mineras y agroalimentarias. Del mismo modo se está observando una disposición -al menos en el discurso- por parte de los actores gubernamentales, quienes se están abriendo a la incorporación, a través de distintos instrumentos de gestión territorial como son las estrategias de desarrollo regional y comunal, de una política que tienda a potenciar actividades más amigables con el entorno y en donde es posible potenciar actividades más sustentables y sostenibles en lo ambiental, económico y social. 


\section{Capítulo II}

Siendo coherente con lo formulado, los cambios descritos se verán reflejados en diversas acciones que tanto los actores gubernamentales como privados han iniciado en aquellas regiones con alta valoración en términos de lo que son sus ventajas comparativas. En efecto, nuestra área de estudio se inscribe claramente como un territorio que está viviendo este proceso de transformación productiva. Es así como, cuando examinamos los instrumentos de gestión territorial como son las Estrategias de Desarrollo Regional de los Ríos (2009) y Los Lagos (2009), como de las comunas vinculadas espacialmente al Parque Nacional Puyehue (PNP), como son Lago Ranco, Río Bueno, Puyehue y Puerto Octay, en todas ellas encontramos un factor común, cual es el reconocer en el turismo, asociados a intereses especiales, a uno de los grandes motores de desarrollo para estos espacios geográficos. Del mismo modo, también es posible observar un interés por parte de los privados de llevar a cabo emprendimientos turísticos bajo las modalidades del ecoturismo, agroturismo y etnoturismo. Sin embargo, las acciones muestran iniciativas disímiles, es decir, la lógica de los empresarios grandes están muy por sobre lo que son los emprendimientos de los medianos, pequeños y micro empresarios. Es por esta razón que para poder dimensionar esta situación, nos concentraremos en este capítulo en identificar y caracterizar a estos micro y pequeños emprendedores formales, localizados en las áreas con mayor conectividad y accesibilidad al PNP, como es la localidad de Entre Lagos y las rutas que se extienden desde este centro urbano, de acuerdo a lo indicado por el Ministerio de Obras Públicas (2013): Entre Lagos - Mantilhue (T-985), Entre Lagos - Anticura (CH-215) y Entre Lagos - El Encanto (U-475).
Sobre la base de esta afirmación, se realizó un trabajo de levantamiento de información, tanto secundaria como primaria, la cual arrojó como resultados situaciones disímiles entre la información oficial versus la información recogida en terreno. En efecto, en el primer caso, el Servicio Nacional de Turismo (SERNATUR) de la Provincia de Osorno, registra un total de 39 emprendimientos formales. Respecto de la segunda situación, nos encontramos que al margen de lo informado por SERNATUR, fue posible pesquisar en terreno 48 emprendimientos más, los cuales no están registrados por esta institución por ser de naturaleza informal. En consecuencia, para poder compatibilizar ambos registros, se tomará en consideración un universo de 87 emprendimientos, los cuales, en términos relativos, se distribuyen en $44,8 \%$ formales y un $56,2 \%$ informales.

En atención a lo anterior, la distribución espacial pormenorizada de los emprendimientos totales, tanto formales como informales, se presentan en la Tabla 1.

Tabla 1: Distribución espacial de emprendimientos formales e informales (2014)

\begin{tabular}{|l|c|c|}
\hline \multicolumn{1}{|c|}{ Localidad } & Formales & Informales \\
\hline Anticura & 9 & 9 \\
\hline El Encanto & 1 & 8 \\
\hline Mantilhue & 0 & 7 \\
\hline Entre Lagos & 29 & 24 \\
\hline Total & 39 & 48 \\
\hline
\end{tabular}

Fuente: SERNATUR Provincia de Osorno/ Trabajo de campo (2014). 
Hemos estimado pertinente en el presente estudio registrar tanto los datos de emprendimientos formales como informales, por cuanto si bien la metodología para analizar la competitividad solo es aplicable a datos formales, no podemos dejar fuera los otros, dado el alto peso relativo que estos tienen en la unidad de estudio.

Por otra parte, debemos consignar que todas las metodologías que miden niveles de competitividad están diseñadas en función de la formalidad, hecho que se confirma a través de las instituciones públicas, para quienes las iniciativas informales "no existen" por no estar formalizadas, quedando por lo tanto desamparadas ante la acción pública. Por consiguiente, ante esta realidad, el tratamiento de la información tendrá dos instancias. La primera, para las empresas formales, la metodología propuesta se aplicó a una muestra de empresas formales, quedando las informales fuera de este análisis cuantitativo, pero no cualitativo. Para tal efecto, y como una manera de minimizar este problema, dado a que en la práctica no podemos desconocer su existencia, se optó por la aplicación de entrevistas semi-estructuradas a una muestra de los emprendimientos informales, como asimismo, a algunos actores claves del ámbito del turismo, con el objeto de poder identificar algunas razones que nos aporten pistas respecto a su actual condición.

Para la obtención de esta información se aplicó al universo de empresas formales un Muestreo Aleatorio Espacial Simple (Suarez y Tapia, 2014), obteniéndose como resultado un total de 29 emprendimientos, correspondiente al 74\% del total. Los tipos de servicios predominantes se distribuyen en: 11 de alimentación y 18 de alojamiento.
Para el caso de los de emprendimientos informales (48), se aplicó un muestreo igual al anterior, obteniéndose como resultado una muestra de 20 , equivalente al $41,7 \%$ del total. El motivo del por qué se empleó un criterio muestral distinto, obedece a que los informales presentan un alto grado de homogeneidad que no justificaba estadísticamente el trabajar con un porcentaje superior (ver Tabla 2).

Tabla 2: Distribución de la Muestra de Emprendimientos Formales e Informales en el PNP y el Área de Influencia.

\begin{tabular}{|l|c|c|c|}
\hline Localidad/ Eje Vial & Formal & Informal & Total \\
\hline Entre Lagos & 22 & 9 & 46 \\
\hline Entre Lagos - Mantilhue & 0 & 5 & 7 \\
\hline Entre Lagos - Anticura & 6 & 2 & 14 \\
\hline Entre Lagos - El Encanto & 1 & 4 & 1 \\
\hline Total & 29 & 20 & 68 \\
\hline
\end{tabular}

Fuente: SERNATUR Provincia de Osorno/ Trabajo de campo (2014).

Para identificar y caracterizar los tipos de emprendimientos (formales e informales), también existen varios tipos de clasificaciones. Sin embargo, con el objeto de poder establecer un patrón regular se optó por utilizar la tipología establecida por SERNATUR (2014), la cual consigna en su clasificación 7 grandes áreas, desagregadas en varios tipos de servicios. Con esto, es posible precisar con mayor exactitud el tipo de servicio ofertado, como así también el tipo de categoría que presentan (ver Cuadro 1). 
Cuadro 1: Tipología de servicios turísticos según SERNATUR 2014

\begin{tabular}{|c|c|}
\hline Alojamiento & $\begin{array}{l}\text { Albergue o refugio, alojamiento fa- } \\
\text { miliar, Apart hotel, camping, lodge, } \\
\text { complejo turístico o resort, depar- } \\
\text { tamento, hacienda o estancia, hos- } \\
\text { pedaje rural, hostal, hostería, hotel, } \\
\text { motel o cabaña. }\end{array}$ \\
\hline Alimentación & $\begin{array}{l}\text { Restaurantes o similares: Estable- } \\
\text { cimientos que prestan servicios de } \\
\text { expendio de comidas y bebidas a la } \\
\text { mesa y/o mostrador, para consumo } \\
\text { en el mismo }\end{array}$ \\
\hline Agencias y ТT.OO. & $\begin{array}{l}\text { Agencias de viajes y/o tour operado- } \\
\text { res: La agencia de viajes actúa como } \\
\text { intermediario entre el proveedor de } \\
\text { servicios y/o tour operador y el usua- } \\
\text { rio final o cliente, entregándole ase- } \\
\text { soría para la planificación y compra } \\
\text { de su viaje. }\end{array}$ \\
\hline Transporte & $\begin{array}{l}\text { Comprende a las empresas que rea- } \\
\text { lizan traslado de pasajeros ya sea } \\
\text { por carretera, aeropuerto, taxi, rent a } \\
\text { car, barco, ferrocarril. }\end{array}$ \\
\hline Esparcimiento & $\begin{array}{l}\text { Establecimientos dedicados a la re- } \\
\text { creación, diversión y entretenimien- } \\
\text { to. }\end{array}$ \\
\hline Guías de Turismo & $\begin{array}{l}\text { Guías de turismo general, guía espe- } \\
\text { cializada, Guía de turismo local, Guía } \\
\text { de turismo de sitio, Guía shuttle o } \\
\text { transferible. }\end{array}$ \\
\hline Turismo Aventura & $\begin{array}{l}\text { Turismo en que se realizan activida- } \\
\text { des específicas que utilizan el en- } \\
\text { torno o medio natural como soporte } \\
\text { físico y recurso para producir en los } \\
\text { turistas determinadas emociones y } \\
\text { sensaciones de descubrimiento y } \\
\text { de exploración, y que implican cier- } \\
\text { to empeño, actividad física y riesgo } \\
\text { controlado. }\end{array}$ \\
\hline
\end{tabular}

Fuente: SERNATUR 2014
Establecidos los tipos de emprendimientos formales (muestra), se procedió a identificarlos y georeferenciarlos, según el tipo de servicios, para cada una de las localidades en las cuales están presentes (ver figuras 1, 2 y 3).

Figura 1: Localización de emprendimientos formales según tipos de servicios en la localidad de Entre Lagos.

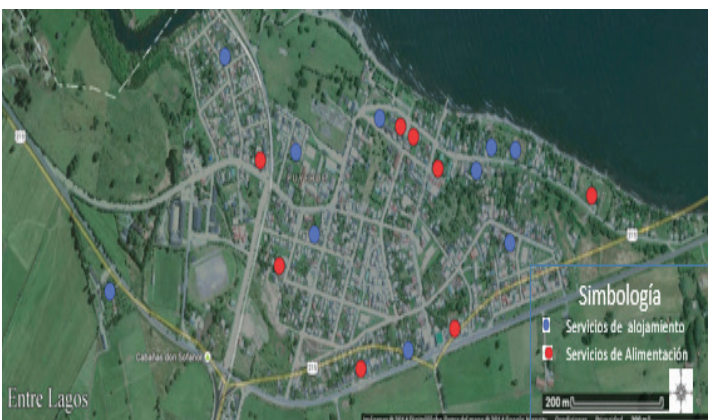

Fuente mapa base: Google Maps. Diseño: Elaboración propia

En este contexto debemos indicar que, en esta georeferenciación fueron exclu-idos los emprendimientos informales, en virtud de la alta movilidad espacial que presentan de año en año.

Figura 2: Localización de emprendimientos formales en área de influencia de Entre Lagos

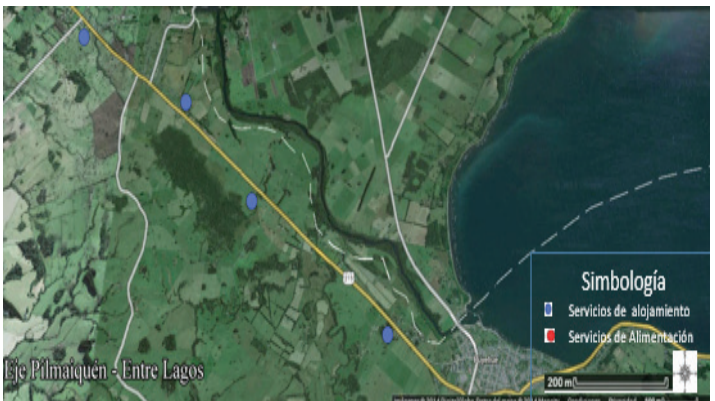

Fuente mapa base: Google Maps. Diseño: Elaboración propia 
Figura 3: Localización de emprendimientos formales en los ejes Entre Lagos-Anticura (Ruta CH-215) y Entre Lagos-El Encanto (Ruta HH-34).

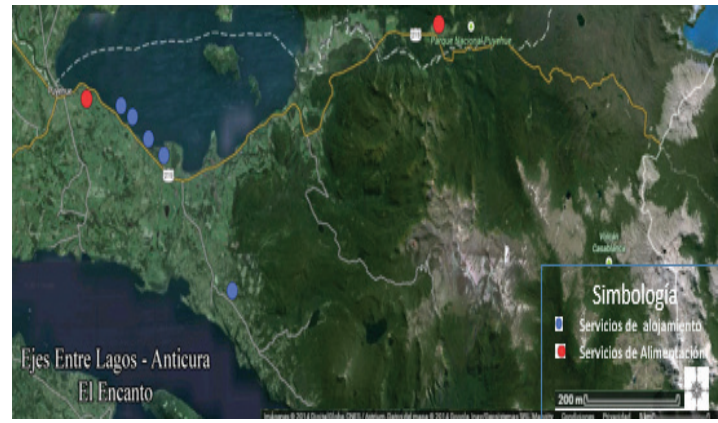

Fuente mapa base: Google Maps. Diseño: Elaboración propia

Una primera observación, nos deja en evidencia que de todas las unidades espaciales a ser estudiadas, la única que dejaremos fuera en este primer análisis es el eje Entre Lagos - Mantilhue, por contar solamente con emprendimientos informales. En consecuencia, la distribución espacial con mayor concentración está dada por Entre Lagos y su área más próxima, Pilmaiquén, seguidos por el eje Entre Lagos - Anticura y finalmente el eje Entre Lagos - El Encanto.

La caracterización de las MIPYMES, en las unidades citadas, nos permitió en primer lugar identificar 22 emprendimientos formales en la localidad de Entre Lagos y su área periférica, distribuidos en 14 servicios de alojamiento y 8 de alimentación. En el eje Entre Lagos - Anticura, se detectaron 6 emprendimientos, siendo 4 de alojamiento y 2 de alimentación. Finalmente, en el eje Entre Lagos - El Encanto se detectó 1 emprendimiento de alojamiento.

De acuerdo a lo señalado, podemos concluir que en toda la unidad espacial la cifra alcanzó un total de 18 servicios de alojamiento y 11 servicios de alimentación. Del mismo modo, como era dable esperar, la Localidad de Entre Lagos es la que concentra la mayor cantidad de empresas, algo natural considerando que es el principal centro urbano de la comuna, además de encontrarse en todo el área de estudio con una localización espacial privilegiada, equidistante tanto de la ciudad capital de la provincia (Osorno), como también del paso internacional Cardenal Samoré, en ambos casos conectada por la ruta internacional $\mathrm{CH}-215$. Aprovechando esta misma ruta, el eje Entre Lagos - Anticura también se ha visto enormemente favorecido, no así la ruta hacia el Encanto, que si bien se inicia en Entre Lagos, para llegar a dicho destino se debe seguir una ruta secundaria, de menor importancia en cuanto al flujo vial.

En suma, podemos confirmar a partir de estos primeros resultados, que existe por parte de estos emprendedores formales una valoración en lo que respecta a localizarse en un centro poblado o próximo a él, como lo es Entre Lagos, como asimismo, en aquellos circuitos con la mayor accesibilidad y conectividad hacia el PNP, como es la ruta internacional $\mathrm{CH}-215$, debido al flujo permanente que presenta, el cual se ve fuertemente incrementado durante el periodo estival.

\section{Capítulo III}

En la actualidad, es un tanto difícil poder realizar una medición de la competitividad a nivel empresarial, puesto que los modelos conocidos están orientados principalmente hacia cálculos dirigidos a países, regiones o sectores productivos en específico, dejando en segundo plano la evaluación para micro, pequeñas y 
medianas empresas localizadas espacialmente en áreas rurales.

Por lo anterior, se optó por aplicar la metodología propuesta por Castaño y Gutiérrez (2011), que cumple con ambos requisitos para esta investigación, es decir, determinar el nivel de competitividad de microempresarios localizados en ámbitos rurales.

\subsection{Funcionamiento del Modelo}

El modelo utilizado funciona sobre la base de ocho factores que poseen una ponderación $(P)$ predefinida dentro del Modelo. Cada factor tiene indicadores (I), también con una ponderación predefinida, que constituyen la base para su evaluación, como lo muestra el Cuadro 2. Cada Factor presenta un nivel de gestión que se calcula algebraicamente de la siguiente manera:

(1) $\quad F G=P F G * S I F G$

(2) $F A d m=P F A d m * S$ IFAdm

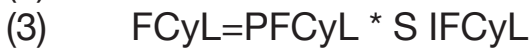

(4) $\quad F F=P F F * S ~ I F F$

(5) $\mathrm{TH}=\mathrm{PTH} * \mathrm{~S}$ ITH

(6) $\quad \mathrm{FT}=\mathrm{PFT} * \mathrm{~S}$ IFT

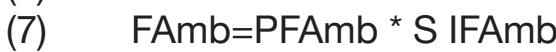

(8) $\quad \mathrm{FE}=\mathrm{PFE} * \mathrm{~S}$ IFE

\section{Donde:}

$\mathrm{FG}=$ Función Gerencial

FAdm = Función Administrativa

FCyL = Función Comercial y Logística

$\mathrm{FF}=$ Función Financiera

$\mathrm{TH}=$ Talento Humano

$\mathrm{FT}=$ Función Tecnológica

FAmb = Función Ambiental

$\mathrm{FE}=$ Función Externa
Cuadro 2: Resumen de Factores con sus Indicadores y ponderaciones tanto para cada indicador, como para cada Factor

\begin{tabular}{|c|c|c|c|}
\hline FACTORES & EKDICADORES & 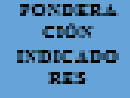 & $\begin{array}{l}\text { TONDER A } \\
\text { CtOX } \\
\text { TACTORES }\end{array}$ \\
\hline \multirow{3}{*}{$\begin{array}{l}\text { Funciän } \\
\text { gereneial }\end{array}$} & + Nivel etueativo genentea. & 40 & \multirow{3}{*}{11,4} \\
\hline & +Experientia & 15 & \\
\hline & Toma de dexisioner. & 35 & \\
\hline TOTAL & & 100 & \\
\hline \multirow{3}{*}{$\begin{array}{l}\text { Funciän } \\
\text { adminiatrativa }\end{array}$} & - Dlaneacián eutratigiza & 35 & \multirow{3}{*}{13,6} \\
\hline & + Extrusture argenimecienal & 35 & \\
\hline & + Norma legplex y tributaria & 30 & \\
\hline TOTAL & & 100 & \multirow{15}{*}{21,2} \\
\hline \multirow{14}{*}{$\begin{array}{l}\text { Funciän } \\
\text { camercial y } \\
\text { lagintica }\end{array}$} & + Krea Cemereial ertablesida. & 7 & \\
\hline & + Expartasionen. & 8 & \\
\hline & + Mersendsa y Ventux. & 8 & \\
\hline & + Experientis en el mereada. & 4 & \\
\hline & + Censcinienta de competidarer. & 5 & \\
\hline & + Qeatiba Proverdosez. & 8 & \\
\hline & + Geatib́= Pradueta. & 8 & \\
\hline & + Qsatiba Presio. & 7 & \\
\hline & + Qratiba Dlara o Distrikusibe & 8 & \\
\hline & + Qsatib́= Premscién. & 8 & \\
\hline & + Senvisia al elients. & 8 & \\
\hline & +Alienmu extratégjens. & 5 & \\
\hline & + Caliabd total. & 6 & \\
\hline & + Invertigecien \& Derarrallo. & 6 & \\
\hline TOTAL & & 100 & \\
\hline \multirow{4}{*}{$\begin{array}{l}\text { Funciän } \\
\text { financitra }\end{array}$} & + Rentabilidad. & 30 & \multirow{4}{*}{13,2} \\
\hline & , Endesdamiznts. & 25 & \\
\hline & +Adninistrecién finmeisra. & 25 & \\
\hline & 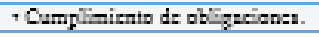 & 20 & \\
\hline TOTAL & & 100 & \\
\hline \multirow{5}{*}{ Talcate humano } & + Culture argenizucianal. & 25 & \multirow{5}{*}{13,7} \\
\hline & + Mentja del idiona inglén. & 15 & \\
\hline & - Fapositarián y peomosiba & 25 & \\
\hline & + Salud y xeguridad iadeatrial. & 20 & \\
\hline & +Evaluasión dal dexemgetho. & 15 & \\
\hline TOTAL & & 100 & \\
\hline \multirow{3}{*}{$\begin{array}{l}\text { Funciän } \\
\text { tecanalógica }\end{array}$} & + Mequuiastiz y equipas. & 31,83 & \multirow{3}{*}{9,3} \\
\hline & + Inuavazión Tesnalagien. & 39,39 & \\
\hline & +Vigilaneia Trealógiza. & 39,39 & \\
\hline TOTAL & & 100 & \\
\hline $\begin{array}{l}\text { Funciän } \\
\text { ambicatal }\end{array}$ & $\begin{array}{l}\text { Doiltien ambiental da la } \\
\text { engreas }\end{array}$ & 10 & 7,6 \\
\hline TOTAL & & 100 & \\
\hline \multirow{5}{*}{$\begin{array}{l}\text { Factarea } \\
\text { externon }\end{array}$} & + Sitrasib́n scanimites del pair. & 20 & \multirow{5}{*}{8,5} \\
\hline & +Cambior teenalógiean. & 20 & \\
\hline & + Fastarer poilticsu y legialativor. & 20 & \\
\hline & + Fastarea asciscaituralen. & 20 & \\
\hline & -Faztarea intemscianalea & 20 & \\
\hline TOTAL & & 100 & 100 \\
\hline
\end{tabular}

Fuente: Modelo de Competitividad de Castaño y Gutiérrez (2011) 
De acuerdo al nivel de gestión de las organizaciones, en cada uno de los factores considerados es posible detectar fortalezas y debilidades en cada factor evaluado, de acuerdo a lo que señala la Figura 4.

Figura 4: Nivel de Gestión de los Factores

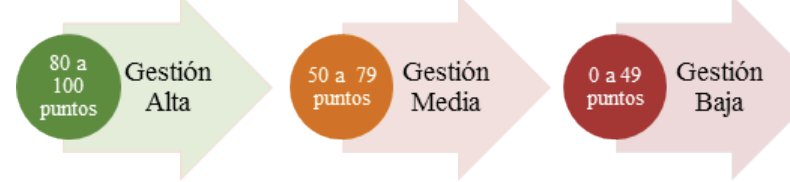

Fuente: Castaño y Gutiérrez, 2011

Finalmente, la expresión que permite calcular el Nivel de Competitividad de la Empresa (NCE) es la siguiente:

$$
\mathrm{NCE}=\frac{\mathbf{F G}+\mathbf{F A d m}+\mathbf{F C y L}+\mathbf{F F}+\mathbf{T H}+\mathbf{F T}+\mathbf{F A m b}+\mathbf{F E}}{100}
$$

El cálculo del NCE arroja un puntaje, donde el máximo que puede obtener una empresa respecto a su nivel de competitividad es 100 puntos. A partir de este valor, se construye una escala que permite ubicar a la empresa en un rango de competitividad y le proporcionará una idea global de la situación en la que se encuentra frente a sus competidores, tal cual se expresa en la Figura 5.
Figura 5: Niveles de competitividad de una empresa

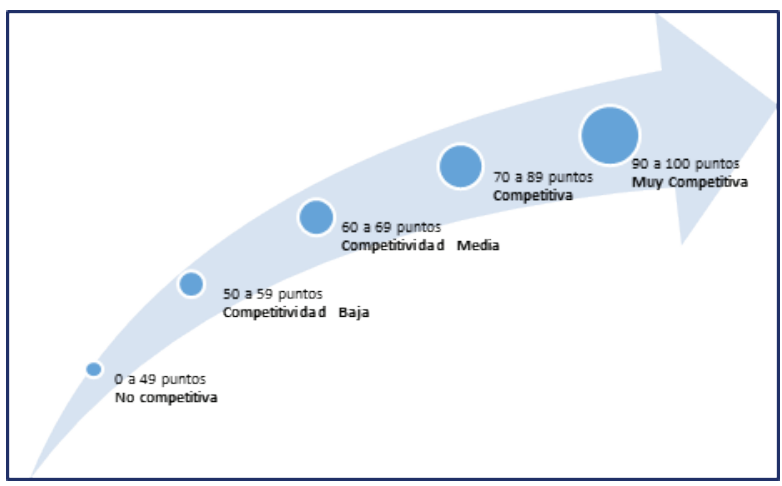

Fuente: Castaño y Gutiérrez, 2011

\subsection{Resultados emprendimientos formales}

\subsubsection{Resultados para cada Factor del Modelo}

Siguiendo el orden establecido por el modelo, se presentan los resultados obtenidos factor por factor. Debemos dejar presente que el modelo pondera con un mayor porcentaje aquellos factores que afectan más directamente a estos emprendedores, relativizando en consecuencia a aquellos que son de carácter más general y pertenecientes a ámbitos más vinculados con la macro-economía.

\section{Factor Función Gerencial.}

Trata de la formación de quienes manejan las actividades delasempresasyelaprovechamiento de las posibilidades materiales y humanas, su conocimiento y experiencia, y cómo esto influye en su capacidad de tomar decisiones. 
Figura 6: Porcentaje de empresas según niveles de gestión en función gerencial
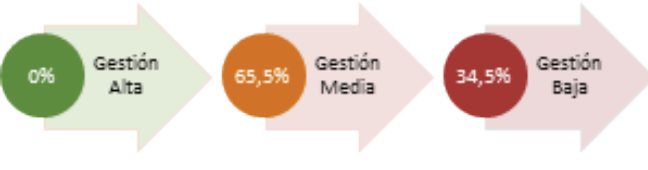

Fuente: Elaboración propia

\section{Factor Función administrativa}

Tiene relación con la capacidad de organizar y administrar los procesos dentro del negocio y el trabajo, de acuerdo a elementos importantes que dan paso a planear objetivos y metas. Una estructura dentro de la organización permite comunicar, motivar, medir, desarrollar y formar personas.

Figura 7: Porcentaje de empresas según niveles de gestión en función administrativa

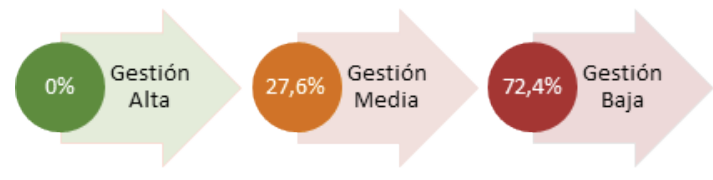

Fuente: Elaboración propia

\section{Factor Función comercial y logística}

Se basa en la logística integral, que consiste en un conjunto de técnicas y medios utilizados para gestionar los flujos de materiales e información, con objeto de maximizar la satisfacción al cliente y minimizar los tiempos de respuestas y costos. El área comercial cobra importancia por el conocimiento del mercado en el cual se desenvuelve la empresa, destacando sus ventajas propias y aprovechando las debilidades de los competidores.

Figura 8: Porcentaje de empresas según niveles de gestión en función comercial y logística
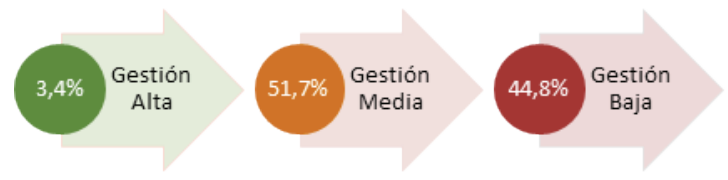

Fuente: Elaboración propia

\section{Factor Función financiera}

Se obtiene información acerca del óptimo control y manejo de los recursos económicos y financieros de la empresa, que aseguren un correcto funcionamiento y la consecución de los objetivos y metas estipuladas.

Figura 9: Porcentaje de empresas según niveles de gestión en función financiera

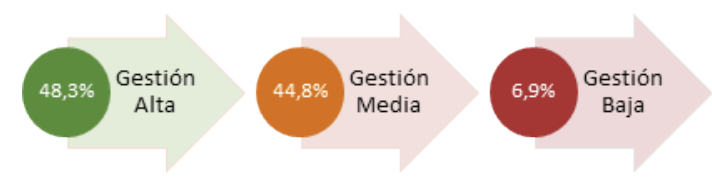

Fuente: Elaboración propia

\section{Factor Talento humano}

Se refiere al ambiente organizacional y la cultura creada dentro de la institución: la satisfacción de los propios trabajadores, a través de programas de desarrollo intra-empresarial, y la evaluación del desempeño de cada uno de ellos, en un ambiente cohesionado en pro de la obtención de lograr resultados más satisfactorios. 
Figura 10: Porcentaje de empresas según niveles de gestión en talento humano
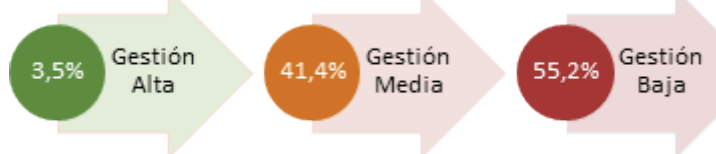

Fuente: Elaboración propia

\section{Factor Función tecnológica}

Tiene relación con el uso y mantenimiento de maquinarias y equipos utilizados a diario dentro de la organización, además de preparación de posibles innovaciones en materia tecnológica y la adaptación a estos cambios.

Figura 11: Porcentaje de empresas según niveles de gestión en función tecnológica

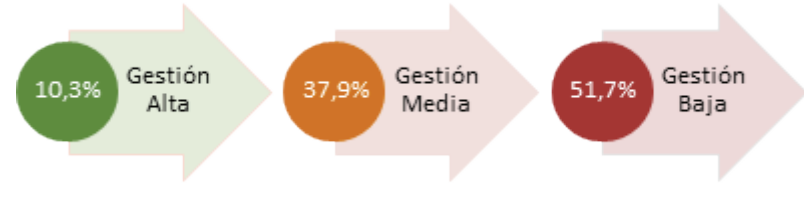

Fuente: Elaboración propia

\section{Factor Función ambiental}

Indica el grado de compromiso de la empresa con políticas ambientales, lo que involucra tanto el respeto hacia normas y procedimientos relacionados con el medio ambiente, como las estrategias utilizadas para su cumplimiento y control.
Figura 12: Porcentaje de empresas según niveles de gestión en función ambiental

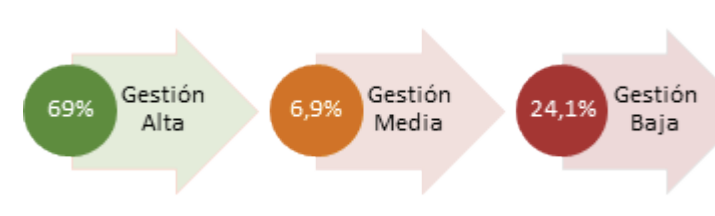

Fuente: Elaboración propia

\section{Factor Factores externos}

Este ítem arroja información relevante de cómo las empresas se preparan para cambios que pueden afectar su mercado objetivo, ya sea económicos, tecnológicos, políticos, legislativos o socio-culturales, sean estos en el ámbito nacional o internacional.

Figura 13: Porcentaje de empresas según niveles de gestión en factores externos

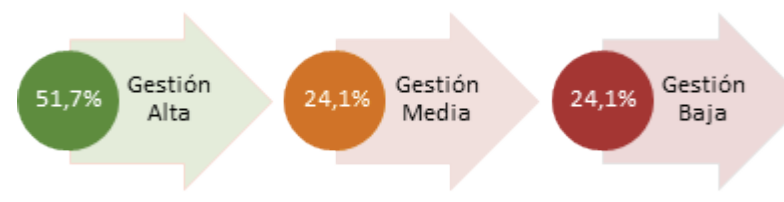

Fuente: Elaboración propia

\subsubsection{Resultados del Nivel de Competitividad de las Empresas (NCE)}

En suma, del análisis realizado para cada uno de los factores que contempla el modelo, podemos destacar que los factores relacionados con la función Gestión Administrativa (72,4\%), Talento Humano $(55,2 \%)$ y Tecnológica $(51,7 \%)$, son las que están determinando la mayor baja en cuanto a la competitividad de estos emprendedores clasificados como mipymes turísticas en nuestra 
área de estudio. Esta situación se confirma al observar la Figura 14, donde, agrupados todos los factores, se llega a establecer un porcentaje de un $62 \%$ si sumamos los que presentan ninguna y baja competitividad. Por lo tanto, se confirma que más allá de constituirse en los emprendedores con la mejor localización geográfica en todo el sistema asociado al PNP, por ahora están haciendo uso principalmente de lo que son sus claras ventajas comparativas, quedando por ahora las ventajas competitivas en una situación secundaria.

Figura 14: Nivel de Competitividad de las empresas analizadas

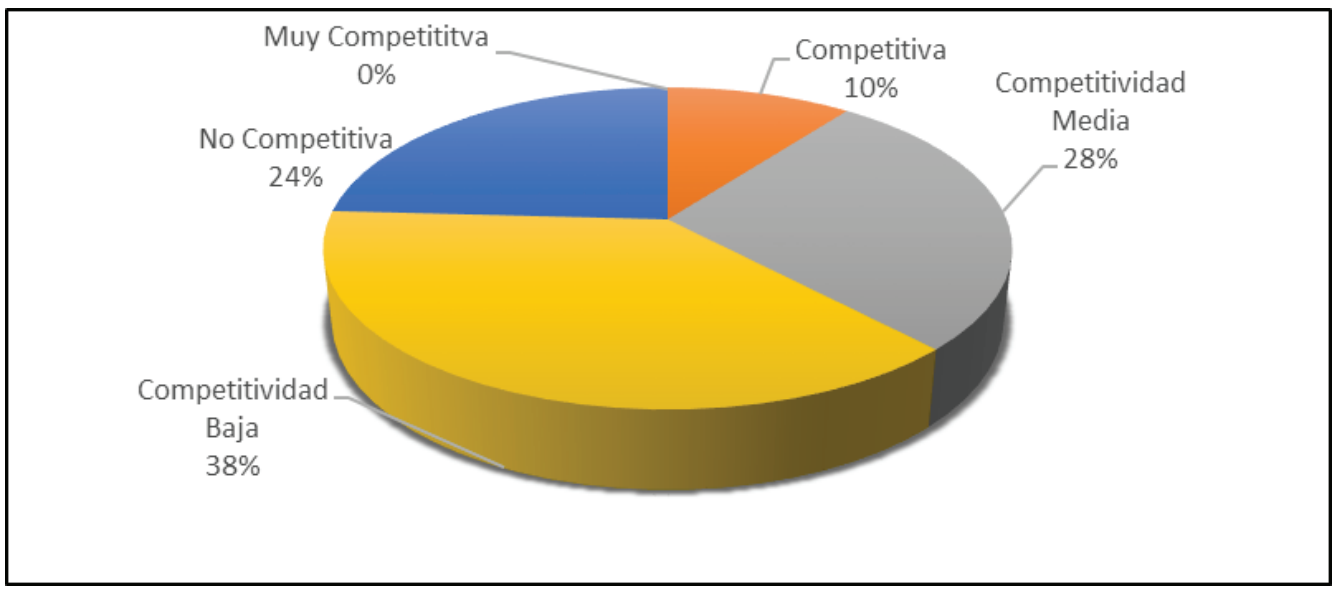

Fuente: Elaboración propia

\subsection{Análisis empresas informales}

Como se indicó al comienzo de esta investigación, hemos tenido que incorporar en este análisis, de manera complementaria, a los emprendimientos informales, producto del gran número encontrado, el cual supera en términos tanto absolutos como relativos a los emprendedores formales. En tal sentido, la información obtenida nos permite afirmar que estas se concentran principalmente en la localidad de Entre Lagos, con un 64\%, seguido de un 23\% en el Entre Lagos - Mantilhue. Esta última, pertenece a la comuna de Rio Bueno, siendo la totalidad de las empresas encuestadas en este lugar de naturaleza informal. Los servicios ofertados en este eje son en su totalidad para camping, arriendo de piezas y kioscos tipo bazar. Además, presentan una alta temporalidad, supeditado esencialmente a los cortos meses del periodo estival.

En los restantes ejes fue posible apreciar iniciativas muy incipientes relacionadas con servicios que estaban desaparecidos en las empresas formales, tales como servicios de 
esparcimiento, como cabalgatas, turismo rural y senderismo a ciertos sectores del PNP. Además, se apreciaron también, especialmente en el eje Entre Lagos - el Encanto, esfuerzos por realizar un etnoturismo, aprovechando la presencia de comunidades originarias, como Mawi-Danche y Calfuco, quienes están intentando poner en valor su patrimonio cultural expresado en la parte alimentaria, artesanal, relación con el entorno, entre otros.

En cuanto al sistema de organización laboral, se encontró un bajo número de personas por emprendimiento. La capacidad de trabajadores de forma permanente, en promedio, fue de 2 personas por empresa, y en forma temporal solo 1, los cuales en su mayoría corresponde al sexo femenino. En cuanto al nivel educacional, el $52 \%$ manifestó tener educación media, un $32 \%$ técnica, un $13 \%$ básica y solo un $5 \%$ educación superior.

También fue posible determinar el nivel de experiencia en la actividad que desarrollaban. Si bien un 77\% manifestó tener experiencia y conocimiento del rubro turístico, con más de 5 años en el sector, se aprecia que la experiencia no va acompañada con un mejoramiento en la gestión. Gran parte de lo que ellos ofertan está destinado a turistas extranjeros, y sin embargo, el manejo del idioma inglés es deficiente. En efecto, un $71 \%$ indicó que su conocimiento es muy básico y el $29 \%$ restante no entiende nada.

Otro aspecto detectado en estos emprendedores informales, corresponde a que el mayor volumen de turistas que demandan sus servicios pertenecen al segmento de jóvenes o adultosjóvenes, que gustan de la aventura al aire libre, ya sea en cabalgatas, tracking, senderismo, escaladas, tirolesas, entre otros, quedando en consecuencia los segmentos etáreos mayores interesados en otros destinos que le ofrezcan mayor calidad y sobre todo comodidad en el servicio.

En cuanto a si realizan algún tipo de capacitación para mejorar la oferta y los servicios, indicaron que sí lo tienen presente, pero lamentablemente es muy difícil para ellos acceder a ello, por cuanto la mayoría de los cursos se realizan durante la temporada alta, más aún cuando cuentan con un personal mínimo para delegar en un tercero su quehacer. Entre los cursos que se les han ofertado destacan: atención al cliente, administración de empresa y contabilidad, inglés, computación, gastronomía, artesanía y tejido, higiene y manipulación de alimentos, primeros auxilios y servicios hoteleros, entre otros.

Las entidades que más colaboran en incentivar el desarrollo del turismo en el territorio, han focalizado su esfuerzo para que ellos se formalicen. Es así como SERNATUR, Indap, Fosis y las respectivas municipalidades de Rio Bueno y Entre Lagos están diseñando acciones que vayan en esta dirección. Lo mismo ocurre con otras instituciones públicas (aunque en menor grado) como Corfo, Sence y Sercotec. En este sentido, los emprendedores de los Ríos (eje Entre Lagos - Mantilhue) valoran de mejor modo a estas instituciones que la de los Lagos.

Se apreció también una situación distinta en cuanto a los que cuentan con accesibilidad al parque, respecto de aquellos que no la tienen. En promedio, las notas más altas son asignadas a las empresas familiares que utilizan el entorno del parque como potencial de su negocio, es decir, aquellos que entregan servicios de cabalgatas, tracking, senderismo y turismo de intereses especiales en general. En cambio, las 
notas más bajas vienen principalmente del eje Entre Lagos - Mantilhue, respecto de actividades ligadas a alojamiento y alimentación. En lo particular, estos emprendedores informales no ven un potencial beneficio en el PNP, fundamentalmente por la precaria conectividad que tienen con esta área protegida. Este hecho contrasta con los localizados en el eje Entre Lagos - Anticura y Entre Lagos - El Encanto, que sí lo valoran, dada la buena conectividad y accesibilidad a dicho parque nacional.

Finalmente, los reclamos más importantes provienen de los ejes Mantilhue y el Encanto. Estos se traducen en la necesidad de mejorar y aumentar la señalética, en ambos casos, y mejorar el asfalto en algunos sectores del eje el Encanto. La retroalimentación para mejorar su gestión lo hacen de forma verbal, a través de conversaciones con los turistas, quienes les manifiestan los problemas que han tenido, tanto para llegar a sus destinos como también respecto al servicio ofertado. A lo anterior, se suma la mala promoción que existe de los servicios. La publicidad se realiza básicamente a través de letreros ubicados en lugares estratégicos en la ruta, como asimismo mediante folletería y mensajes en radios locales. En suma, concuerdan que lo más necesario para mejorar sus emprendimientos pasan, en orden de importancia, por una mayor publicidad, mayor apoyo estatal, mejor conectividad y mejor tecnología. Esta solicitud, como es posible apreciar, no dista mucho en términos generales de las demandas que los emprendedores formales también manifestaron, tal cual arrojó el modelo aplicado.

\section{Conclusiones}

Al concluir la presente investigación podemos destacar siguiendo el orden establecido en cada uno de los capítulos lo siguiente:

1.- El análisis temporo-espacial nos ilustra, a partir de los cambios que el país vivió desde fines de la década de los setenta y con mayor fuerza en los ochenta, al menos dos situaciones. Primero, queda en evidencia que las importantes transformaciones en materia económica cumplieron con el objetivo de dinamizar y reactivar el país sobre la base de la diversificación y la apertura a los mercados internacionales, al menos durante las dos primeras centurias. Segundo, esta situación comienza a cambiar gradualmente desde fines de la década de los noventa, donde la economía comienza a contraerse y empieza a presentar las primeras fatigas, especialmente en cuanto a las escasas muestras de transitar hacia una segunda fase exportadora, generando efectos socio-espaciales importantes, especialmente en aquellas regiones del país menos competitivas.

2.- En cuanto a la situación particular de explorar actividades económicas de menor impacto ambiental, el turismo aparece como una alternativa con mejor recepción por parte de los distintos actores sociales. Esto se confirma con el alto número de emprendimientos tanto formales como informales identificados en elárea de estudio, donde la mayor concentración se encuentra en la propia localidad de Entre Lagos y la ruta internacional $\mathrm{CH}-215$. Sin embargo, cuando examinamos la lógica de operación, no dista mucho de lo que ya veníamos observando en otras actividades productivas de nuestro país. En efecto, las ventajas comparativas asociadas a las potencialidades del emplazamiento 
escénico, son altamente valoradas por estos emprendedores. En consecuencia, el patrón de vinculación con el entorno de éstos siguen el patrón tradicional, es decir, observan el paisaje y luego se plantean qué hacer; y no cómo lo realizan las sociedades más avanzadas en estas materias, donde el razonamiento es al revés: se preguntan cuáles son las herramientas y el conocimiento, para luego relacionarse con el entorno. Este es el primer indicio del alto número de pequeños emprendedores que presentan un conocimiento basado fundamentalmente en la praxis, más que en sus capacidades y estudios para abordar una iniciación de actividades como las relatadas en este estudio.

3.- Respecto a la aplicación del modelo para medir el nivel de competitividad de estas mipymes turísticas, se confirma la situación citada precedentemente, en donde aquellos factores internos asociados a la función administrativa, la función talento humano y la función tecnológica principalmente, están determinando y condicionando su actual y precaria situación para desarrollar en buenos términos su actividad. Esta situación, complementada con el análisis realizado a los emprendedores informales, no hace más que agravar la situación de los primeros. En efecto, al no estar formalizados, generan una competencia desleal que termina finalmente desmotivando a quienes han hecho un esfuerzo por invertir y ofertar servicios de mejor calidad.

En suma, se espera con el desarrollo de este temario, aproximarnos a confirmar que la sola explotación de las ventajas comparativas presentes en nuestra área de estudio son insuficientes para explicar la situación inestable e incierta que afecta a estos micro y pequeños emprendedores turísticos, quienes han visto a través de esta actividad una oportunidad para salir de las precarias condiciones en las cuales actualmente se encuentran. De allí que resulta plausible comprender por qué esta actividad es solo temporal y con escaso valor agregado, hecho que les impide dar ese salto cualitativo que les permita romper la condición de fragilidad presente y transitar hacia un desarrollo más sostenible en lo social, económico y ambiental.

\section{Bibliografía}

Aristóteles. 1991. La Política. México: Ed. Porruá.

Benko, G. y Lipietz, A. 1992. Les Régions qui gagnent. Districts et réseaux: les noveaux paradigmes de la geographie économique. París: Presses Universitaires de France.

Castaño, L. y Gutiérrez, A. 2011. Propuesta para determinar la competitividad en las empresas del sector comercial del Área Metropolitana Centro Occidente AMCO. Pereira: Universidad Tecnológica de Pereira.

Gobierno Región de Los Lagos. 2009. Estrategia de desarrollo Región de Los Lagos 2009 - 2020. Puerto Montt: Grafica Andina Ltda.

Gobierno Región de Los Ríos. 2009. Estrategia de desarrollo Región de Los Ríos 2009 - 2019. Valdivia: Trama Impresores S.A.

Haguenauer, L. 1990. "La competitividad: una reseña bibliográfica con énfasis en el caso brasileño". Pensamiento Iberoamericano n¹7. Madrid: Sociedad Estatal Quinto Centenario. Krugman, P. 1992. Geografía y Comercio. Barcelona: Antoni Bosch Editor.

Ministerio de Obras Públicas. 2013. Red Caminera de Chile. Carta Caminera 2013 (formato digital). Disponible en: http://www. mapas.mop.cl/cc2013/6-red-caminera-chile-los-rios-los-lagos.jpg Observatorio europeo Leader. 1997. "Innovación y desarrollo rural”. Serie Informes del Observatorio N². Bruselas, Bélgica.

Porter, M. 1991. La Ventaja Competitiva de las Naciones. Madrid: Plaza y Janés.

President's commission on industrial competitiveness. 1985. Global competition: a new reality. Washington DC: PCIC.

Real Academia Española. 2014. Diccionario de la Lengua 
Española. Madrid: RAE.

Ricardo, D. 1973 [1817]. On the Principles of Political Economy and Taxation (Edited by Piero Sraffa, with the collaboration of M. H. Dobb). Cambridge: Cambridge University Press.

Rosales, C. 1990. Valorización de los factores productivos a través de las empresas exportadoras de uva de mesa en la región semiárida chilena: hacia una explicación de los contrastes entre el Limarí y el Choapa. Santiago: Facultad de Arquitectura y Urbanismo, Universidad de Chile.

SERNATUR. 2011. Chile, Estrategia nacional de turismo 20122020. Santiago: SERNATUR.
SERNATUR. 2014. Buscador de sitios turísticos. Santiago: SERNATUR. Disponible en: http://www.sernatur.cl/buscador-deservicios-turisticos

Smith, A. 1999 [1776]. Investigación sobre la naturaleza y causa de la riqueza de las naciones. México: Fondo de Cultura Económica.

Suarez, M. y Tapia, F. 2014. Interaprendizaje de estadística básica. Ibarra, Ecuador: Universidad Técnica del Norte.

Szary, A.-L. 1997. "Regiones ganadoras y regiones perdedoras en el retorno de la democracia en Chile: poderes locales y desequilibrios territoriales". Revista EURE, 23(70): 59-78. 
